\title{
Suppression of RND3 activity by AES downregulation promotes cancer cell proliferation and invasion
}

\author{
HONGWEI XIA ${ }^{1 *}$, MINGXING LI ${ }^{*}$, LIANG CHEN ${ }^{1}$, WEIBING LENG ${ }^{2}$, DANDAN YUAN $^{2}$, \\ XIAOHUI PANG ${ }^{2}$, LIU CHEN ${ }^{2}$, RONGHUI LI ${ }^{2}$, QIULIN TANG ${ }^{2}$ and FENG BI ${ }^{1,2}$ \\ ${ }^{1}$ Laboratory of Signal Transduction and Molecular Targeted Therapy, State Key Laboratory \\ of Biotherapy, West China Hospital, Sichuan University; ${ }^{2}$ Department of Medical Oncology, \\ West China Hospital, Sichuan University, Sichuan, Chengdu 610041, P.R. China
}

Received November 20, 2012; Accepted January 2, 2013

DOI: $10.3892 / \mathrm{ijmm} .2013 .1321$

\begin{abstract}
Amino-terminal enhancer of split (AES) is a member of the Groucho/TLE family. Although it has no DNA-binding site, AES can regulate transcriptional activity by interacting with transcriptional factors. Emerging evidence indicates that AES may play an important role in tumor metastasis, but the molecular mechanism is still poorly understood. In this study, we found that knockdown of AES by RNA interference (RNAi) downregulated RND3 expression at the mRNA and protein levels in MDA-MB-231 and HepG2, two cancer cell lines. Furthermore, luciferase assays showed that overexpression of AES significantly enhanced RND3 promoter activity. Moreover, inhibition of AES both in MDA-MB-231 and HepG2 cells by RNAi significantly promoted cell proliferation, cell cycle progression and invasion, consistent with the effects of RNAi-mediated RND3 knockdown in these cells. For the first time, data are presented showing that alteration of the malignant behavior of cancer cells by AES is related to RND3 regulation, and these findings also provide new insights into the mechanism of AES action in regulating tumor malignancy.
\end{abstract}

Correspondence to: Dr Feng Bi, Laboratory of Signal Transduction and Molecular Targeted Therapy/Department of Medical Oncology, West China Hospital, Sichuan University, No. 37 Wai Nan Guoxue Road, Sichuan, Chengdu 610041, P.R. China E-mail: bifeng@medmail.com.cn

${ }^{*}$ Contributed equally

Abbreviations: AES, amino-terminal enhancer of split; TLE, transducin-like enhancer of split; TFs, transcription factors; RNAi, RNA interference; CRC, colorectal cancer; NICD, Notch intercellular domain; RhoGAP, GTPase-activating protein for Rho; ROCK, Rho kinase-I; HCC, hepatocellular carcinoma; ESCC, esophageal squamous cell carcinoma

Key words: AES, RND3, cancer cells, proliferation, invasion

\section{Introduction}

The majority of patients with malignant carcinoma succumb to metastasis (1), and inhibition of cancer cell metastasis is an effective method to prolong the survival time of patients. Yet, the mechanisms by which tumor cells become metastatic are poorly understood (2). Thus, to explore the molecular mechanisms and molecular targets of metastasis is of vital importance in the targeted therapy of cancer (3).

Amino-terminal enhancer of split (AES), which is known as Groucho (Gro) or transducin-like enhancer of split (TLE), is a member of the transcriptional co-repressor family (4). The Gro/TLE protein family consists of five members and is highly conserved within different species (5). These proteins have no DNA-binding region but can interact with DNA-binding transcription factors (TFs) and mediate transcription activity of target genes (6,7). AES, as well as its mouse homolog GRG5, is the shortest member of the Gro/TLE family $(4,8)$. It was considered that AES may act as a negative regulator in the process of transcription. Moreover, a series of previous research described that AES recruits various TFs such as NF- $\kappa B$ and gp130 to gene promoters, and functions as a transcription repressor $(9,10)$. Most of the previous studies concerning AES, as well as other members of the Gro/TLE family, have mainly focused on development, including bone growth, hematopoiesis and eye development (11-14), while there are only a few studies concerning the function of AES in cancer. AES also forms complexes with the mitochondrial protein Bit1 and induces cell death with characteristics of caspase-independent apoptosis (15). Recently, AES was reported as a metastatic repressor in colorectal cancer (CRC) by inhibiting the NOTCH signaling pathway, and composite depletion of AES in Apc intestinal polyposis mice caused marked tumor invasion and intravasation $(16,17)$. Thus, loss of AES may be an important factor for inducing tumor invasion. Although AES is known to regulate the activity of the Notch intercellular domain (NICD), other pathways involved in AES regulation during the process of tumor metastasis must be explored.

The Rho GTPase family is a group of small GTPase proteins that are implicated in several cellular functions such as actin cytoskeleton organization, microtubule dynamics and cell cycle progression. Thus, they play an important role in 
cancer progression (18). Most Rho GTPase members function as molecular switches, cycling between an active GTP-bound and an inactive GDP-bound conformation (19). RND3, also known as RhoE, is an atypical member of the Rho GTPase family in that it binds, but does not hydrolyze GTP. The bestcharacterized function of RND3 is inhibition of RhoA/ROCK signaling by binding to p190 GTPase-activating protein for Rho (RhoGAP), thus reducing the RhoA-GTP levels, as well as directly regulating the RhoA effector, Rho kinase-I (ROCK-I) (20). Recent studies have indicated that RND3 could acts as a tumor-suppressor gene in cancer progression $(21,22)$. However, the molecular mechanism that regulates the expression of RND3 is poorly understood.

Herein, the results revealed that reduced AES expression downregulates RND3 expression at the mRNA and protein levels, while enhanced AES expression significantly increases the activity of the RND3 promoter. Further studies have shown that AES regulates tumor cell proliferation, cell cycle progression and invasion, and the process is related to RND3 regulation. For the first time, we demonstrated that AES regulates the expression of RND3, and the results further elucidate the mechanisms of AES in regulating tumor malignancies.

\section{Materials and methods}

Cell culture. The human hepatocellular carcinoma cell line HepG2 and the breast cancer cell line MDA-MB-231 were maintained in RPMI-1640 medium (Invitrogen, Gaithersburg, MD, USA) supplemented with $10 \%$ heat-inactivated fetal bovine serum (FBS) (Gibco, Gaithersburg, MD, USA), 100 U/ml of penicillin $\mathrm{G}$ sodium, and $100 \mu \mathrm{g} / \mathrm{ml}$ streptomycin sulfate (Sigma, St. Louis, MO, USA) in a humidified atmosphere containing $5 \% \mathrm{CO}_{2}$ at $37^{\circ} \mathrm{C}$.

Plasmid construct. The human AES gene (NM_001130, 197 amino acids) was PCR amplified from human HEK293 cDNA. To construct HA-AES, the AES coding sequence was amplified by sense primer 5'-GCCCCGAATTCCGATTGACATGA-3' and anti-sense primer 5'-GAACGGTACCCCCTGCTAATC CGACTTCTCGCCAT-3' and then cloned into the EcoR1 and Kpn1 sites of the pCMV-HA vector. RND3 promoter was cloned into the pGL3-basic vector to generate the pGL3RND3-promoter as previous described (23).

Small interfering RNA transfection. The siRNAs against AES were designed and synthesized by Ribobio (Guangzhou, China). The sequence of AES siRNA was 5'-CCUACGG CUUGAACAUCGAdTdT-3' and the sequence of the RND3 siRNA strand was 5'-AACAGATTGGAGCAGCTACdTdT-3'. siRNAs against AES and RND3 were transfected into MDA-MA-231 and HepG2 cells respectively using Lipofectamine 2000 (Invitrogen).

Luciferase reporter assay. The pGL3-RND3-promoter $(0.4 \mu \mathrm{g})$ and pRL-TK $(0.005 \mu \mathrm{g})$ were co-transfected into HeLa cells in each well of 24-well plates together with pCMV-HA or pCMVHA-AES. Firefly and Renilla luciferase activities were measured $48 \mathrm{~h}$ after transfection using the Dual-Luciferase reporter assay system (Promega) according to the manufacturer's protocol.
Values are shown as relative Renilla and Firefly luciferase activity, and data values are reported as means \pm SEM.

$R T-P C R$. Total RNAs were extracted from transfected cells by TRIzol (Life Technologies) using the manufacturer's protocol and reconstituted in $1.0 \mu \mathrm{g} / \mu \mathrm{l}$ with nuclease-free water. For semi-quantitative reverse transcription-PCR, cDNA was synthesized from total RNA using Olig-dT primer. The primers used for specific RND3 PCR reactions were: forward 5'-AAGATA GTTGTGGTGGGAGA-3' and reverse 5'-CATAGTAAGGAGA ACCCGAA-3'. The specific AES PCR reaction primers used were: forward 5'-CACCAGGAGGATGATGGCGAG-3' and reverse 5'-GGCGTGGAGGTGTCTGGAACTA-3'. The primers used for specific GAPDH PCR reactions were: forward 5'-CAA GGCCAACCGCGAGAA-3' and reverse 5'-CCCTCGTAGAT GGGCACAGT-3'.

Western blotting. Transfected cells were lysed in RIPA buffer (150 mM NaCl, 1\% NP-40, 50 mM Tris- $\mathrm{HCl} \mathrm{pH}$ 7.4, $1 \mathrm{mM}$ phenylmethylsulfonyl fluoride, $1 \mu \mathrm{g} / \mathrm{ml}$ leupeptin, $1 \mathrm{mM}$ deoxycholic acid and $1 \mathrm{mM}$ EDTA) containing a cocktail of protease inhibitors and phosphatase inhibitors (Calbiochem, Darmstadt, Germany). Equal amounts of the protein sample (30-50 $\mu \mathrm{g})$ were separated by $12 \%$ SDS-PAGE and transferred to PVDF membranes (Millipore, Bedford, MA, USA) using the Bio-Rad semi-dry transfer system. The following antibodies were used for western blotting: anti-AES (Sigma), anti- $\alpha$-tubulin (Biostar, Wuhan, China), anti-RND3 (Millipore).

Cell proliferation assay. MDA-MB-231 and HepG2 cells were seeded in 96-well plates, and after $24 \mathrm{~h}$, cells were transfected with two types of siRNAs or the negative control, respectively. Relative cell growth was measured using the Cell Counting Kit-8 (Dojingdo, Kumamoto, Japan).

Matrigel invasion assay. After $24 \mathrm{~h}$ of transfection, cells were collected and suspended in serum-free medium. Cells $\left(4 \times 10^{4}\right)$ in $0.2 \mathrm{ml}$ serum-free medium were plated in the top chamber with a Matrigel-coated membrane (24-well insert; pore size, $8 \mathrm{~mm}$; Becton Dickinson), with $10 \%$ FBS as a attractant. The cells were incubated for $48 \mathrm{~h}$. The cells that did not invade through the pores were removed, and the filter was stained with hematoxylin and eosin (H\&E) for visualization and counting.

Cell cycle analysis by flow cytometry. Cells were transfected with siRNA against AES or RND3 for $48 \mathrm{~h}$. The cells were then digested by trypsin, collected by centrifugation, washed with PBS and fixed overnight at $4^{\circ} \mathrm{C}$ by $70 \%$ ethanol. The cells were the washed with PBS and stained by PI at $4^{\circ} \mathrm{C}$ for $30 \mathrm{~min}$ using the Cell Cycle Detection kit (KeyGen, Nanjin, China). The cells were then analyzed using a flow cytometer (BD FACSCalibur).

Statistical analysis. The data are presented as the means \pm SEM. The Student's t-test was used for comparisons. $\mathrm{p}<0.05$ was considered to indicate a statistically significant result.

\section{Results}

siRNA-mediated AES knockdown downregulates RND3 expression at the $m R N A$ and protein levels. Previous studies 
A
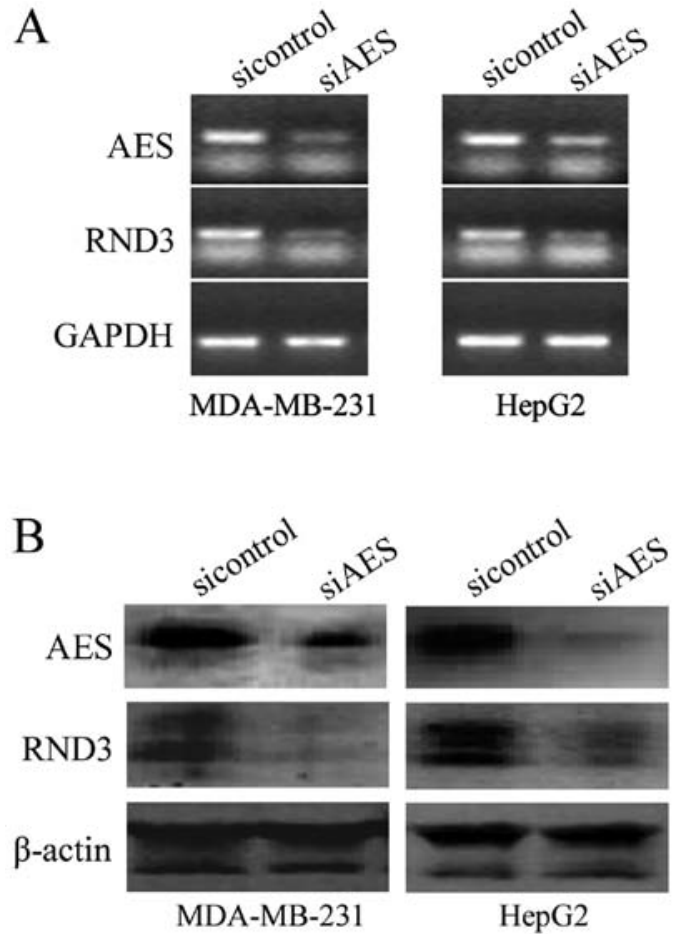

Figure 1. AES regulates the expression of RND3. (A) The mRNA expression level of AES and RND3 in MDA-MB-231 and HepG2 was analyzed by RT-PCR. GAPDH was used as a loading control. (B) Immunoblot analysis of AES and RND3 expression in MDA-MB-231 and HepG2 cells transfected with sicontrol or siAES. $\beta$-actin was used as a loading control.

reported that AES acted as a potential tumor-metastasis suppressor $(16,17)$. However, the molecular mechanisms remain unclear (17). RND3, a typical member of the Rho family, was demonstrated as a tumor-suppressor in several studies $(14,24)$. Recent studies indicate that both AES and RND3 are involved in tumor metastasis. Thus, we hypothesized that AES could regulate RND3 expression levels in cancer cells. Breast cancer cell line MDA-MB-231 and hepatocellular carcinoma cell (HCC) line HepG2 were transfected with AES siRNA. RT-PCR results revealed that knockdown of AES by RNA interference (RNAi) downregulated RND3 expression at the mRNA level (Fig. 1A). Western blot analysis showed that downregulation of AES also inhibited RND3 expression at the protein level in both the MDA-MB-231 and HepG2 cell lines (Fig. 1B). These results reveal that AES regulates RND3 at the mRNA and protein levels.

Enhanced AES expression increases RND3 promoter activity. The relationship between AES and RND3 was further investigated. To investigate whether AES regulates RND3 promoter activity, the promoter sequence of RND3 was subcloned into the pGL3-basic vector. Then, the pGL3-RND3-promoter was co-transfected with pCMV-HA-AES or pCMV-HA, respectively, into HeLa cells. Dual-luciferase reporter assay revealed that forced AES expression increased the luciferase activity of the pGL3-RND3-promoter by 5.6-fold compared with the control pCMV-HA group. Meanwhile, AES siRNA abolished the activation of luciferase activity induced by enhanced AES expression (Fig. 2). In summary, these data indicate that AES functions as a positive regulator of the RND3 promoter.

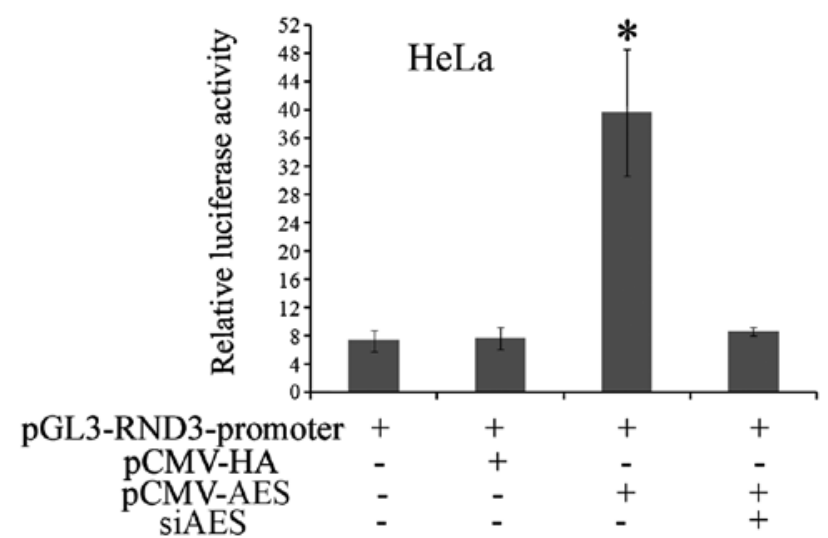

Figure 2. AES regulates RND3 promoter activity. pGL3-RND3-promoter luciferase constructs and the pRL-TK reporter plasmid were cotransfected into HeLa cells with pCMV-HA, pCMV-AES or RND3 siRNA, respectively. Luciferase activity was determined $48 \mathrm{~h}$ after transfection. The pRL-TK reporter plasmid was used as the transfection control. The ratio of normalized sensor to control luciferase activity is shown. Data are shown as the means \pm SD and were obtained from three independent experiments performed in triplicate. ${ }^{*} \mathrm{p}<0.05$, a significant difference from the control transfected cells.

Downregulation of AES and RND3 expression enhances the proliferation of cancer cells. Previous studies have shown that RND3 functions as a tumor-suppressor gene in esophageal squamous cell carcinogenesis, and is involved in the regulation of tumor cell proliferation, invasion and cell cycle progression (25-27). Recent research also indicates that AES may have a similar function with RND3 (16). Our results showed that AES regulates the expression of RND3 in breast cancer and HCC cells. Thus, whether AES and RND3 function similarly in these two cancer cell lines needs to be further investigated. To determine the influence of AES and RND3 on tumor proliferation, specific siRNAs of AES and RND3 were separately transfected into MDA-MB-231 and HepG2 cells. After transfection, the viability of both HepG2 and MDA-MB-231 cells was markedly increased at each time point similar to the effect of RND3 knockdown (Fig. 3). This result demonstrated that downregulation of AES promotes cancer cell proliferation.

siRNA-mediated AES and RND3 downregulation induces cell cycle progression of cancer cells. The cell cycle progression of cancer cells with different transfection was assayed by flow cytometry. The percentage of G1 phase cells transfected with AES siRNA was decreased significantly compared with the controls (Fig. 4). Downregulation of AES in both MDA-MB231 and HepG2 cells promoted S-G2-M phase progression, which was in correspondence with the effect of RND3 knockdown by siRNA (Fig. 4). These results indicate that AES also contributed to cell cycle progression and this function involved RND3 regulation.

Knockdown of AES and RND3 increases the invasive activity of cancer cells. We performed a Matrigel invasion assay to investigate the invasive activity of cancer cell lines. MDA-MB-231 and HepG2 cells were transfected with control, AES or RND3 siRNAs separately. Knockdown of both AES and RND3 expression resulted in a significant increase in the 
A

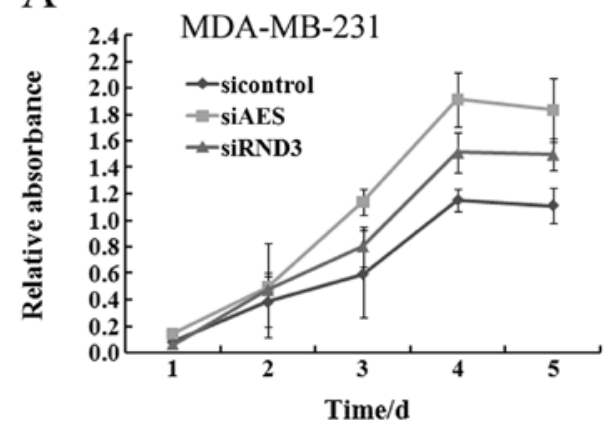

B

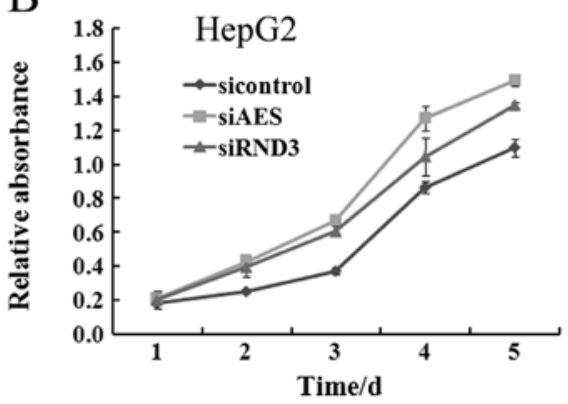

Figure 3. AES influences proliferation potential of cancer cells. Proliferation potential of (A) MDA-MB-231 and (B) HepG2 cells $24 \mathrm{~h}$ after transfection with sicontrol, siAES or siRND3, was determined by the CCK-8 assay. The data represent means \pm SD from 3 independent experiments performed in triplicate.

number of invasive cells compared with the control groups (Fig. 5). This result suggests that downregulation of AES enhances breast cancer and HCC cell invasion by mimicking the inhibition of RND3 expression.

\section{Discussion}

AES, a member of the Gro/TLE family, regulates gene expression at the transcription level by interacting with various transcriptional factors (TFs) (28-31). In the past few years, studies concerning AES as well as other members of the TLE/ GRG family have mainly focused on various developmental and pathological processes (32-34). Recently, Sonoshita et al reported that AES/GRG5 prevents metastasis of colorectal cancer cells by inactivating Notch signaling and may function as a metastasis-suppressor gene, which highlights an innovative therapy for anti-metastasis (16). However, the regulatory mechanism of AES in tumorigenesis and cancer progression remains to be further determined. Our present study suggests that AES-mediated RND3 regulation plays an important role in the process of cell proliferation, cell cycle progression and invasion.

In the present study, small specific siRNA was used to knockdown AES expression in MDA-MB-231 and HepG2 cells, and the results showed that RND3 expression was decreased accompanied by AES downregulation. These data indicate that AES regulates RND3 directly or indirectly. A dual-luciferase reporter assay was used to determine whether AES influences RND3 promoter activity. Forced AES expression significantly activated RND3 promoter activity in HeLa cells. However,
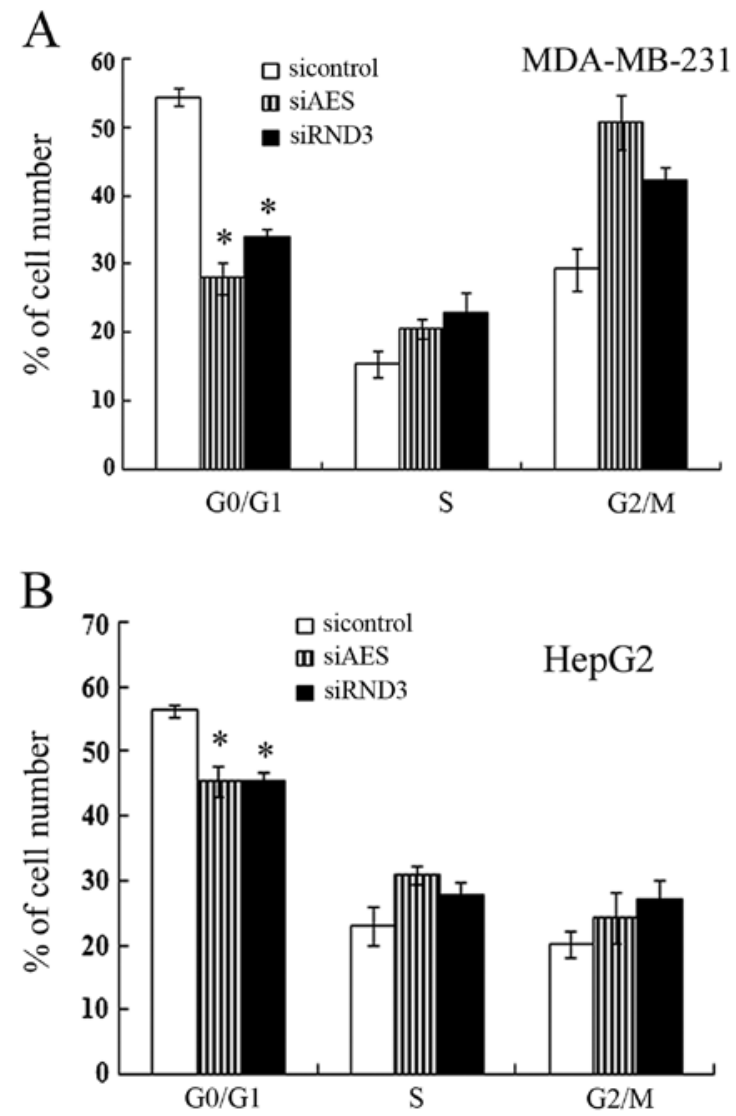

Figure 4. Downregulation of both AES and RND3 promotes cell cycle progression. Cell cycle progression of (A) MDA-MB-231 and (B) HepG2 cells $48 \mathrm{~h}$ after transfection with sicontrol, siAES or siRND3, was determined by FACS analysis. Data are representative of three independent experiments. * $p<0.05$, a significant difference from oligo-transfected control cells.

whether AES interacts with the RND3 promoter directly still requires further investigation.

At the transcriptional level, RND3 may be directly regulated by many TFs, including P53, HIF-1 $\alpha$ and Foxd3 (35-37). Moreover, DNA damage-inducing stimuli, including chemotherapeutic agents and ultraviolet (UV) irradiation, could upregulate the RND3 gene expression at both the mRNA and protein levels (38). According to the characteristics of the Gro/TLE family members, we hypothesized that AES interacts with various TFs and promotes RND3 expression at the transcription level. Therefore, it will be interesting to explore the accurate molecular mechanisms by which AES interacts with TFs and affects RND3 transcription.

RND3 is an atypical member of the Rho family, and studies concerning this molecule are relatively fewer compared with studies of members of the Rho family (39). Previous studies showed that RND3 regulates a diverse set of biological activities including actin organization, cell motility, cell-cycle progression and apoptosis $(24,27)$.

Recent research revealed that elevated RND3 expression markedly increased the expression levels of PTEN and p27, while decreasing pAkt expression, thus inhibiting cell cycle progression at the G1 phase $(26,40)$. RND3 also blocks cell cycle progression at the $\mathrm{G} 2 / \mathrm{M}$ phase. A study using a prostate cancer cell line showed that forced RND3 overexpression inhibits the expression levels of $\mathrm{CDC} 2$ and cyclin $\mathrm{B} 1$ which are essential 
A

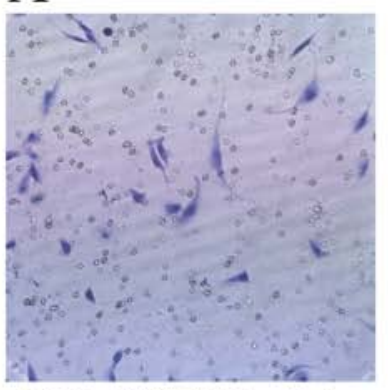

MDA-MB-231/sicontrol

\section{B}

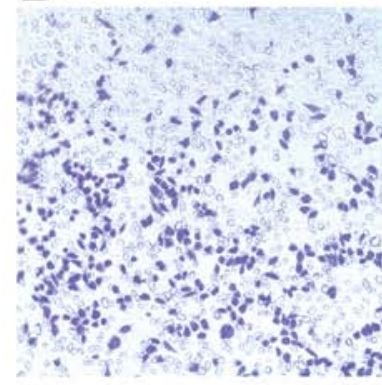

HepG2/sicontrol

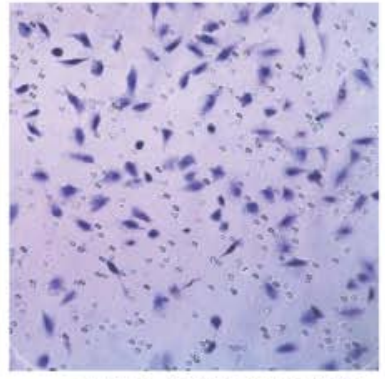

MDA-MB-231/siAES

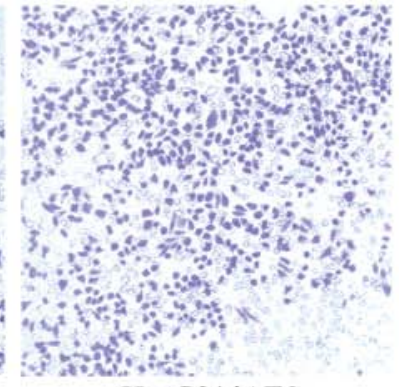

HepG2/siAES

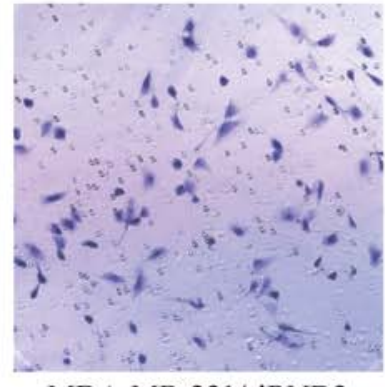

MDA-MB-231/siRND3

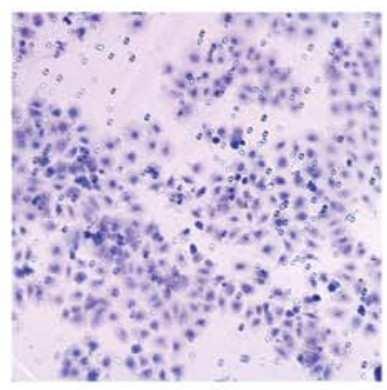

HepG2/siRND3
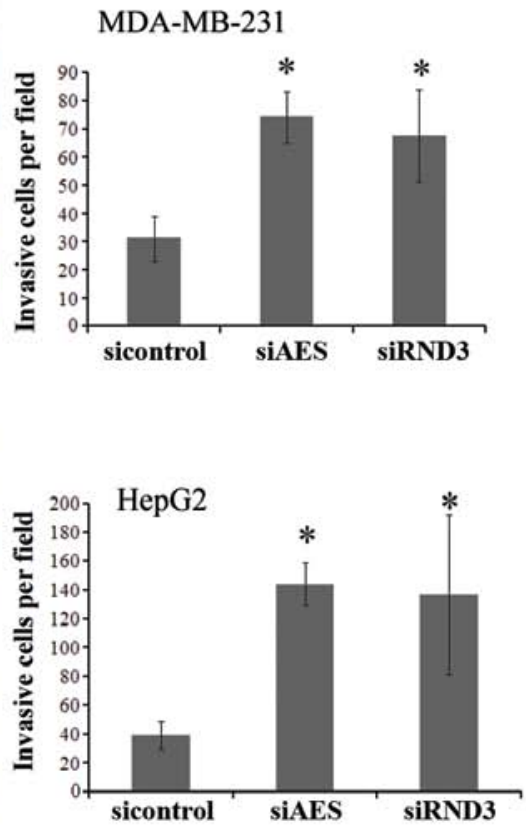

Figure 5. AES influences cancer cell invasion. The invasive ability of (A) MDA-MB-231 and (B) HepG2 cells 48 h after transfected with sicontrol, siAES or siRND3, was assayed using a Matrigel-coated Transwell. The cells that successfully invaded into the Matrigel were quantified 48 h after plating. Data are represented as the means \pm SD for three independent experiments. ${ }^{*} \mathrm{p}<0.05$, a significant difference from the control oligo-transfected cells.

for $\mathrm{G} 2 / \mathrm{M}$ transition, and induces $\mathrm{G} 2 / \mathrm{M}$ phase arrest (41). Moreover, downregulation of RND3 in ESCC cells promoted cell proliferation, cell cycle progression, as well as cell invasion in vitro (26).

Our results revealed that siRNA-mediated AES downregulation decreased the expression of RND3; therefore, we hypothesized that AES executes its function through RND3. Further functional experiments were carried out using these two genes. Rapid proliferation was induced by AES-specific siRNA transfection in both MDA-MB-231 and HepG2 cells. Knockdown of AES in these two cell lines also induced cell cycle progression and promoted cell invasion. These effects were also noted when cells were transfected with RND3specific siRNA and were consistent with the effects of AES-specific siRNA transfection. The above results indicate that AES regulates RND3 expression and suggest that downregulation of AES promotes tumor cell proliferation, cell cycle progression and invasion which involves RND3 expression. We demonstrated, for the first time, that there is a connection between AES and RND3, a typical member of the Rho family. Our results also elucidate the mechanisms of AES regulation and offer new insights into the molecular mechanisms of how AES executes its tumor repressor functions.

\section{Acknowledgements}

This research was supported by the National Natural Science Foundation of China (81071640, 30971519), National Basic Research Program of China (2011CB935800) and International Cooperation and Communication in Science and Technology Project of Sichuan Province (2010HH0006).

\section{References}

1. Gupta GP and Massague J: Cancer metastasis: building a framework. Cell 127: 679-695, 2006.

2. Oppenheimer SB: Cellular basis of cancer metastasis: a review of fundamentals and new advances. Acta Histochem 108: 327-334, 2006.

3. Dolgin E: Cancer metastasis scrutinized. Nature 461: 854-855, 2009.

4. Beagle B and Johnson GV: AES/GRG5: more than just a dominant-negative TLE/GRG family member. Dev Dyn 239: 2795-2805, 2010.

5. Bajoghli B: Evolution of the Groucho/Tle gene family: gene organization and duplication events. Dev Genes Evol 217: 613-618, 2007.

6. Chen $\mathrm{G}$ and Courey AJ: Groucho/TLE family proteins and transcriptional repression. Gene 249: 1-16, 2000.

7. Fisher AL and Caudy M: Groucho proteins: transcriptional corepressors for specific subsets of DNA-binding transcription factors in vertebrates and invertebrates. Gene Dev 12: 1931-1940, 1998.

8. Jennings BH and Ish-Horowicz D: The Groucho/TLE/Grg family of transcriptional co-repressors. Genome Biol 9: 205, 2008.

9. Liu F, Liu Y, Li D, et al: The transcription co-repressor TLE1 interacted with the intracellular region of gpl30 through its Q domain. Mol Cell Biochem 232: 163-167, 2002.

10. Ghosh HS, Spencer JV, Ng B, McBurney MW and Robbins PD: Sirt1 interacts with transducin-like enhancer of split-1 to inhibit nuclear factor kappaB-mediated transcription. Biochem J 408: 105-111, 2007.

11. Swingler TE, Bess KL, Yao J, Stifani S and Jayaraman PS: The proline-rich homeodomain protein recruits members of the Groucho/Transducin-like enhancer of split protein family to co-repress transcription in hematopoietic cells. J Biol Chem 279: 34938-34947, 2004.

12. Wang WF, Wang YG, Reginato AM, Plotkina S, Gridley T and Olsen BR: Growth defect in Grg5 null mice is associated with reduced Ihh signaling in growth plates. Dev Dyn 224: 79-89, 2002.

13. Aghaallaei N, Bajoghli B, Walter I and Czerny T: Duplicated members of the Groucho/Tle gene family in fish. Dev Dyn 234: 143-150, 2005. 
14. Steffen B, Knop M, Bergholz U, et al: AML1/ETO induces self-renewal in hematopoietic progenitor cells via the Grouchorelated amino-terminal AES protein. Blood 117: 4328-4337, 2011.

15. Jan Y, Matter M, Pai JT, et al: A mitochondrial protein, Bit1, mediates apoptosis regulated by integrins and Groucho/TLE corepressors. Cell 116: 751-762, 2004.

16. Sonoshita M, Aoki M, Fuwa H, et al: Suppression of colon cancer metastasis by Aes through inhibition of Notch signaling. Cancer Cell 19: 125-137, 2011.

17. Christofori G: Metastatic colon cancer cells negotiate the intravasation Notch. Cancer Cell 19: 6-8, 2011.

18. Vega FM and Ridley AJ: Rho GTPases in cancer cell biology. FEBS Lett 582: 2093-2101, 2008.

19. Villalonga $P$ and Ridley AJ: Rho GTPases and cell cycle control Growth Factors 24: 159-164, 2006.

20. Wennerberg K, Forget MA, Ellerbroek SM, et al: Rnd proteins function as RhoA antagonists by activating p190 RhoGAP. Curr Biol 13: 1106-1115, 2003

21. Fortier M, Comunale F, Kucharczak J, Blangy A, Charrasse S and Gauthier-Rouviere C: RhoE controls myoblast alignment prior fusion through RhoA and ROCK. Cell Death Differ 15: 1221-1231, 2008

22. Garg R, Riento K, Keep N, Morris JD and Ridley AJ: N-terminusmediated dimerization of ROCK-I is required for RhoE binding and actin reorganization. Biochem J 411: 407-414, 2008.

23. Chen J, Zhou H, Li Q, et al: Epigenetic modification of RhoE expression in gastric cancer cells. Oncol Rep 25: 173-180, 2011.

24. Chardin P: Function and regulation of Rnd proteins. Nat Rev Mol Cell Biol 7: 54-62, 2006.

25. Grise F, Sena S, Bidaud-Meynard A, et al: Rnd3/RhoE is downregulated in hepatocellular carcinoma and controls cellular invasion. Hepatology 55: 1766-1775, 2012.

26. Zhao H, Yang J, Fan T, Li S and Ren X: RhoE functions as a tumor suppressor in esophageal squamous cell carcinoma and modulates the PTEN/PI3K/Akt signaling pathway. Tumour Biol 33: $1363-1374,2012$

27. Klein RM and Aplin AE: Rnd3 regulation of the actin cytoskeleton promotes melanoma migration and invasive outgrowth in three dimensions. Cancer Res 69: 2224-2233, 2009.

28. Rave-Harel N, Miller NL, Givens ML and Mellon PL: The Groucho-related gene family regulates the gonadotropin-releasing hormone gene through interaction with the homeodomain proteins MSX1 and OCT1. J Biol Chem 280: 30975-30983, 2005.
29. Wang W, Wang YG, Reginato AM, et al: Groucho homologue Grg5 interacts with the transcription factor Runx2-Cbfa1 and modulates its activity during postnatal growth in mice. Dev Biol 270: 364-381, 2004

30. Yu X, Li P, Roeder RG and Wang Z: Inhibition of androgen receptor-mediated transcription by amino-terminal enhancer of split. Mol Cell Biol 21: 4614-4625, 2001

31. Turki-Judeh W and Courey AJ: Groucho: a corepressor with instructive roles in development. Curr Top Dev Biol 98: 65-96, 2012.

32. Brinkmeier ML, Potok MA, Cha KB, et al: TCF and Grouchorelated genes influence pituitary growth and development. Mol Endocrinol 17: 2152-2161, 2003.

33. Metzger DE, Gasperowicz M, Otto F, Cross JC, Gradwohl G and Zaret KS: The transcriptional co-repressor Grg3/Tle3 promotes pancreatic endocrine progenitor delamination and $\beta$-cell differentiation. Development 139: 1447-1456, 2012.

34. Orian A, Delrow JJ, Rosales Nieves AE, et al: A Myc-Groucho complex integrates EGF and Notch signaling to regulate neural development. Proc Natl Acad Sci USA 104: 15771-15776, 2007.

35. Ongusaha PP, Kim HG, Boswell SA, et al: RhoE is a pro-survival p53 target gene that inhibits ROCK I-mediated apoptosis in response to genotoxic stress. Curr Biol 16: 2466-2472, 2006.

36. Zhou J, Li K, Gu Y, et al: Transcriptional up-regulation of RhoE by hypoxia-inducible factor (HIF)-1 promotes epithelial to mesenchymal transition of gastric cancer cells during hypoxia. Biochem Biophys Res Commun 415: 348-354, 2011.

37. Katiyar $\mathrm{P}$ and Aplin AE: FOXD3 regulates migration properties and Rnd3 expression in melanoma cells. Mol Cancer Res 9: 545-552, 2011.

38. Boswell SA, Ongusaha PP, Nghiem P and Lee SW: The protective role of a small GTPase RhoE against UVB-induced DNA damage in keratinocytes. J Biol Chem 282: 4850-4858, 2007.

39. Ellenbroek SI and Collard JG: Rho GTPases: functions and association with cancer. Clin Exp Metastasis 24: 657-672, 2007.

40. Klein RM and Higgins PJ: A switch in RND3-RHOA signaling is critical for melanoma cell invasion following mutant-BRAF inhibition. Mol Cancer 10: 114, 2011.

41. Bektic J, Pfeil K, Berger AP, et al: Small G-protein RhoE is underexpressed in prostate cancer and induces cell cycle arrest and apoptosis. Prostate 64: 332-340, 2005. 